\section{The Law of Conservation of Energy}

\section{Giottis Motsanos*}

Trifilon Technologies, Sweden

The law of conservation of energy is an empirical based physical law, which states that energy can neither be created nor be destroyed; it can only be converted into another. As example the EME system transforms from electrical to mechanical and again to electrical energy.

For a closed system like the EME, this means that the total energy is unchanged with time.

The law of conservation of energy can be breached due to the uncertainty principle of Heisenberg; it has to do with "position and momentum".

A concept that is relevant to the mechanics as well as quantum physics is "Angular momentum" or angular velocity which is an important quantity in quantum physics.

The new laws of mechanics based on the object's size and speed, it is different from Newtonian mechanics. Einstein vs. Newton, see formula below:

$$
\frac{M V^{2}}{2} \Leftrightarrow W=F^{*} s
$$

\section{Power Multiplier Machine}

The model is given below in Figure 1.

$$
\begin{aligned}
& \text { Givet } \mathrm{F}_{2}=13 \mathrm{~kg}=130 \mathrm{~N} \\
& \text { Varvtal } \mathrm{n}_{1}=\mathrm{n}_{2}=1480 \mathrm{rpm} \\
& \mathrm{F}_{1}=\mathrm{F}_{2} / 4=32.5 \mathrm{~N} \\
& \mathrm{~L}_{1}=60 \mathrm{~mm}=0.06 \mathrm{~m} \\
& \mathrm{~L}_{2}=15 \mathrm{~mm}=0.015 \mathrm{~m} \\
& \mathrm{G}_{1}=\mathrm{L}_{1} \times \mathrm{F}_{1}(\mathrm{Nm}) \text { Motor } \\
& \mathrm{G}_{2}=\mathrm{L}_{2} \times \mathrm{F}_{2}(\mathrm{Nm}) \text { Generator } \\
& \mathrm{P}=\mathrm{M} \times \mathrm{W}
\end{aligned}
$$

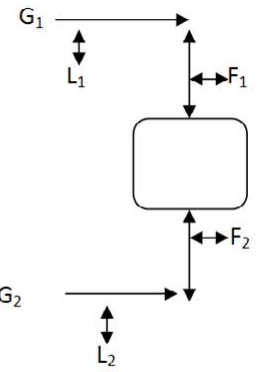

Figure 1: Power multiplier machine model.

$$
\begin{aligned}
& \omega=\frac{2 \times \pi \times r \times n}{60 \mathrm{sec}}=\text { angular velocity } \mathrm{m} / \mathrm{s} \\
& P_{2}=130 \mathrm{~N} \times 2 \times 3.14 \times 0.015 \times 1480=\frac{18133}{60 \mathrm{sec}} \mathrm{Nm}=302 \mathrm{~W} \\
& P_{1}=130 \mathrm{~N} \times 2 \times 3.14 \times 0.06 \times 1480=\frac{18133}{60 \mathrm{sec}} \mathrm{Nm}=302 \mathrm{~W}
\end{aligned}
$$

The workflow efficiency is: input shaft 2.65 times $\times 4$ on output shaft $=10.6$ times.

Total efficiency at $1 \mathrm{Nm} 10.6$ times in the closed inertia system.

The fundamental forces make up 50-60\%, ie gravity and the weak and strong interaction.

Input shaft $\mathrm{P}_{1}=302 \mathrm{~W}$ and output shaft $\mathrm{P}_{2}=302 \times 10.6=3201 \mathrm{~W}$. Prerequisite for this calculation applies to engine and generator of 4 $\mathrm{kW}$ with $80 \%$ efficiency.

According to Lorentz, all inertial systems are equivalent, in the system above differentiala geometry and differential equations are applied.
The difference between Bohr and Einstein is that Bohr wants to count on the unpredictability, which Einstein had trouble with. In EME's "inertial" system can utilize it: 1 . The bounded force of the pulley system. 2. Gravitation. 3. The weak and strong force of alternation.

With the above, and with the support of the Copenhagen interpretation, the EME system's static calculations increase the effectiveness/efficiency of approximately $50-60 \%$ regardless of the configuration of the tool.

PMK has managed to incorporate the macro and the micro cosmos in one unit, because it has a solid frame of reference which distinguishes and control the dependent variable "power" and the independent variable "working distance. Newton had problems with a fixed frame of reference.

Furthermore, the PMK managed to synchronize and harmonize the tool with the help of quantum physics and string theory. In other words marry classical mechanics with quantum mechanics with help of string theory.

Conclusion: PMK can exploit the weak and strong force of alternation that Newton could not. Furthermore, PMK utilize gravity, which quantum physics cannot do practically, much less to incorporate it in its mathematical formula.

Copy Right (: PMK HB 2014.
*Corresponding author: Motsanos G, Founder President at PMK and Marketing Director, Trifilon Technologies, Sweden, Tel: +46 8162000; E-mail: g.motsanos@hotmail.com

Received July 19, 2017; Accepted August 10, 2017; Published August 17, 2017 Citation: Motsanos G (2017) The Law of Conservation of Energy. Fluid Mech Open Acc 4: 172. doi: 10.4172/2476-2296.1000172

Copyright: (C 2017 Motsanos G. This is an open-access article distributed unde the terms of the Creative Commons Attribution License, which permits unrestricted use, distribution, and reproduction in any medium, provided the original author and source are credited. 